\title{
Modélisation de la qualité des eaux des lacs profonds : modèle thermique et biogéochimique du lac du Bourget
}

\author{
Brigitte Vinçon-Leite, Bruno Tassin
}

CERGRENE, Ecole nationale des Ponts et Chaussées, Paris

\section{Introduction}

Le lac du Bourget, d'une surface d'environ $43 \mathrm{~km}^{2}$, est le plus grand lac subalpin situé entièrement en France. Sa profondeur maximale de $145 \mathrm{~m}$ le classe parmi les lacs européens profonds.

La dégradation de la qualité des eaux du lac du Bourget, notable à partir des années 60 a été enrayée par la mise en service, en 1981, d'un ceinturage qui détourne hors du bassin versant l'essentiel des effluents des villes de Chambéry, Aix-les-Bains et le Bourget-du-Lac, soit environ 400000 équivalents-habitants. Cependant, les importants travaux entrepris n'ont pas conduit à toute l'amélioration escomptée de la qualité des eaux.

Une campagne de mesure, complémentaire de l'habituel suivi allégé, a été mise en œuvre (1988-89) avec la collaboration de divers organismes scientifiques ('). Ces données récentes associées à celles du suivi antérieur du lac forment une série de 10 années de mesures consécutives.

S'appuyant sur cette importante base de données, la modélisation thermique et biogéochimique du lac a été entreprise afin de synthétiser les connaissances, de prévoir l'évolution de la qualité des eaux ainsi que l'influence de possibles aménagements complémentaires (ceinturage complet du lac, siphonnage des eaux profondes, ....).

\section{Structure du modèle}

En raison de la profondeur importante du lac du Bourget, de sa nette stratification estivale et du temps de séjour de l'eau ( 7 ans en moyenne), les phénomènes dominants dans le lac surviennent selon l'axe vertical.

L'évolution des variables est décrite par l'équation d'advection-dispersion intégrée dans le plan horizontal :

$$
\begin{aligned}
& \frac{\partial C(z, t)}{\partial t}= \\
& =\frac{1}{A(z)} \frac{\partial}{\partial z}\left[A(z) K(z, t) \frac{\partial C(z, t)}{\partial z}\right] \text { Dispersion } \\
& -\frac{1}{A(z)} \frac{\partial}{\partial z}[A(z) W(z, t) C(z, t)] \quad \text { Transport advectif } \\
& +\operatorname{SS}(z, t) \quad \text { Terme de sources et puits }
\end{aligned}
$$

où $C(z, t)$ est la variable à la profondeur « $z$ » et au temps « $t »$ considérés, $A(z)$ est la superficie de la section horizontale du lac à la profondeur considérée, $K(z, t)$ est le coefficient de dispersion, $W(z, t)$ est la vitesse d'advection de l'eau.

(1) CEMAGREF; Université de Savoie: Université de Genève: Cellule technique du lac du Bourget.

\section{Deep lakes water quality modelling : \\ Thermal and biogeochemical model of Lake Bourget (France)}

In 1981, important works including diversion of the main sewers coming into lake Bourget (one of the largest french alpine lakes) have been implemented. In order to quantify the restoration of the water quality standards, a project including a detailed water quality survey (1988-89) and the thermal and biogeochemical modelling of the lake is undertaken. The model (Temperature, Phosphorus and Oxygen) is one-dimensional vertical. It is based on the advection-diffusion equation. Results of the thermal model agree with the observed seasonal and long-term evolution of temperature in lake Bourget. The results of the biogeochemical sub-model for the calibration phase are presented. 
EVOLUTION DE LA TEMPERATURES A 2 METRES DE 1976 A 1983.

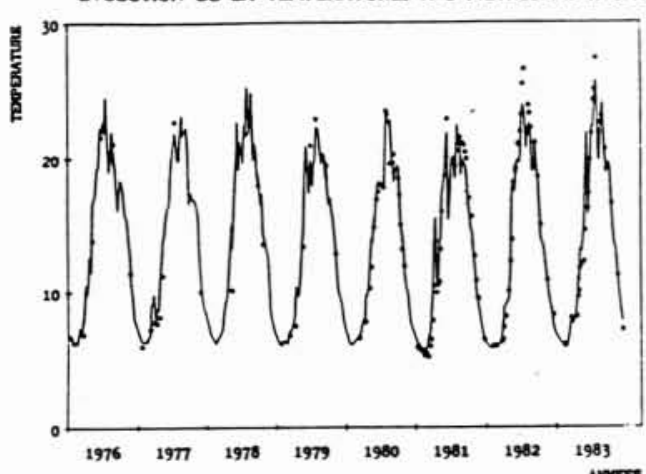

EVOLUTION DE LA TEMPERATURES A 15 METRES DE 1976 A 1983.

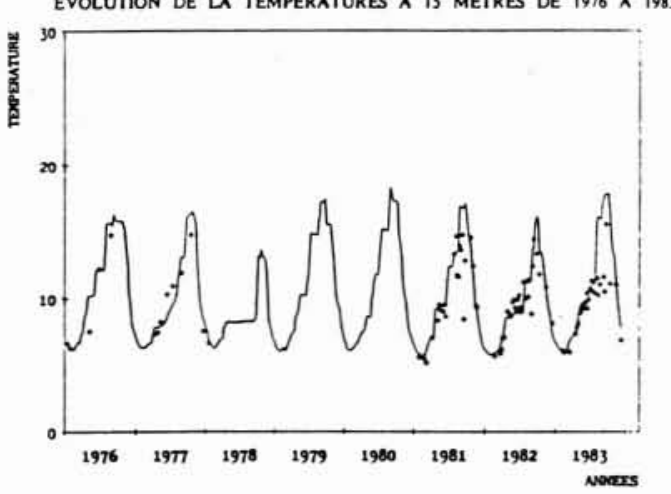

EVOLUTION DE LA TEMPERATURE A 140 METRES DE 1976 A 1983.

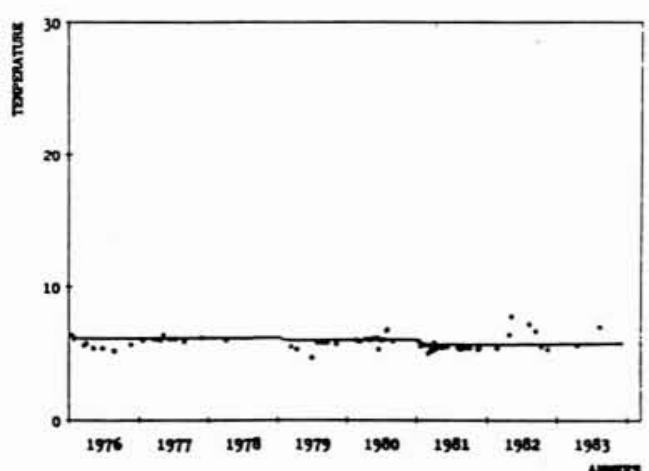

1. Evolution des températures durant la période 1976-1983.

La modélisation comporte tout d'abord une modélisation de la température du lac, qui influe, parmi d'autres facteurs, sur le profil vertical des coefficients de dispersion. Ces profils de dispersion sont utilisés par la suite pour le transport physique des variables physico-chimiques. On retrouve ainsi la prééminence du sous-système physique sur le sous-système écologique (CHAHUNEAU et al., 1980).

Le lac est discrétisé en 145 couches de $1 \mathrm{~m}$ d'épaisseur. Cette épaisseur des couches s'est révélée satisfaisante pour la stabilité de la méthode numérique employée ainsi que pour limiter la diffusion numérique. Sur ce maillage, l'équation est résolue par une méthode aux différences finies semi-implicite non centrée. Le pas de temps de calcul est de $3 \mathrm{~h}$.

\section{Modèle thermique}

\subsection{Formulation de la dispersion verticale}

Le terme de dispersion de l'équation (1) inclut non seulement la dispersion turbulente mais aussi d'autres phénomènes verticaux tels que ceux induits par des hétérogénéités locales du champ de vitesse : circulations de Langmuir, upwellings ou downwellings, ... (СHAHUNEAU et al., 1983).

La paramétrisation du coefficient de dispersion, variable en fonction de la profondeur et du temps, est celle du modèle thermique proposé par TASSIN (1986) pour le lac Lèman.

L'originalité de cette formulation est de suivre l'évolution de la structure thermique du lac: épiliminion, métalimnion et hypolimnion. Durant la période de l'année où il n'y a pas de stratification, la formulation employée dans l'épilimnion reste valable pour tout le lac.

Les équations utilisées sont présentées de manière plus détaillée dans un précédent article (VINÇON-LEITE et al., 1989).

\subsection{Calibration et vérification}

La calibration des paramètres qui interviennent dans le modèle thermique a été réalisée d'après les données de l'année 1981. La vérification du modèle thermique sur une période longue de 8 années comprises entre 1976 et 1983 a montré que le bilan thermique réalisé par le sousmodèle météorologique reste équilibré sur une longue période et que le modèle est numériquement stable.

L'évolution des températures en surface, dans le métalimnion et au fond du lac est représentée sur la figure 1 .

\subsection{Discussion des résultats}

Les résultats présentés montrent un bon accord entre les valeurs calculées et les mesures réalisées dans le lac, en particulier dans l'épilimnion.

Les caractéristiques principales de l'évolution saisonnière des températures dans le lac du Bourget sont dans l'ensemble correctement reproduites :

- établissement de la thermocline au printemps;

- profondeur de l'épilimnion, de la thermocline et du métalimnion ;

- valeur du gradient de température dans le métalimnion ;

- érosion de la thermocline en automne ;

- brassage hivernal des eaux.

\section{Modèle biogéochimique}

Le modèle biogéochimique associé au modèle thermique présenté ci-dessus, simule les cycles du Phosphore et de l'Oxygène. L'objectif du modèle est de représenter, par l'intermédiaire d'un nombre limité de variables, l'évolution saisonnière, et de prévoir à long terme la qualité des eaux du lac. 
La limitation du nombre de variables permet de restreindre le nombre des paramètres à ajuster ainsi que le type de données mesurées nécessaires à la calibration. La transposition ultérieure du modèle à d'autres lacs présentant des caractéristiques analogues en sera donc facilitée.

Les variables du modèle sont les suivantes:

- Orthophosphates.

- Phosphore organique particulaire.

- Oxygène dissous.

Les équations qui décrivent les principaux phénomènes pris en compte par le modèle biogéochimique sont présentées dans le tableau 1 .

Le modèle a été développé en deux étapes, la première concerne le cycle du phosphore et la seconde celui de l'oxygène dissous.

\subsection{Sous-modèle Phosphore}

L'hypothèse de base du sous-modèle Phosphore est de représenter l'évolution de la biomasse phytoplanctonique par celle du Phosphore organique particulaire (IMBODEN, 1974 ; 1978 ; TASSIN, 1987).

L'élément limitant de la production primaire est le Phosphore minéral dissous.

La production primaire (éq. 2) est limitée par :

- la concentration en orthophosphates selon une cinétique de type Monod;

- par la température selon une fonction, croissante jusqu'à la température optimale de la production primaire, présentant un palier de la température optimale à la température maximale et décroissante au-delà de la température maximale;

- par la lumière selon la loi de Steele.

La minéralisation du Phosphore organique particulaire (éq. 3) est influencée seulement par la température selon une loi d'Arrhénius.

La vitesse de sédimentation du Phosphore particulaire est constante dans l'épilimnion et dans l'hypolimnion. Conformément aux observations réalisées dans d'autres lacs stratifiés, la vitesse de sédimentation est plus élevée dans l'hypolimnion (JAQUET, 1983).

Le relargage des orthophosphates (éq. 4) à l'interface eau-sédiment est décrit par une cinétique qui tient compte des concentrations d'oxygène et d'orthophosphates à l'interface (GRIFFIN et FERRARA, 1984; TASSIN, 1987). Les concentrations d'oxygène et d'orthophosphates interviennent selon une cinétique de type Monod inverse qui privilégie l'augmentation rapide du relargage quand l'oxygène diminue et qui le limite quand la concentration en orthophosphates surnageant augmente (TASSIN, 1987).

\subsubsection{Calibration du sous-modèle Phosphore}

Le sous-modèle Phosphore a été calibré d'après les données de forçage et les mesures réalisées durant l'année 1981. Dans cette première phase oủ l'oxygène n'est pas une variable d'état du modèle, les concentrations d'oxygène au fond qui influent sur le processus de relargage des orthophosphates sont fournies comme variables de forçage.

\section{Tableau 1. Equations des processus considérés} dans le sous-modèle biogéochimique.

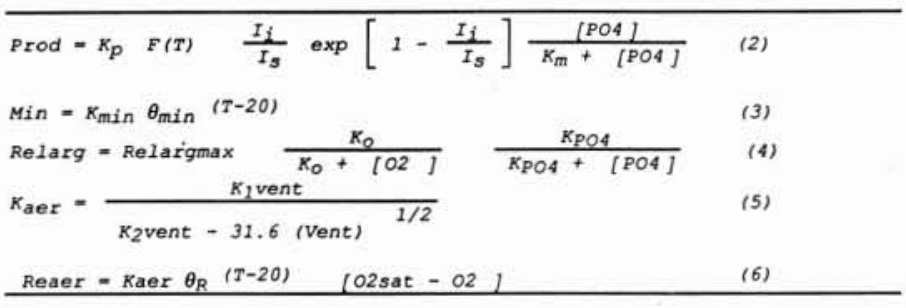

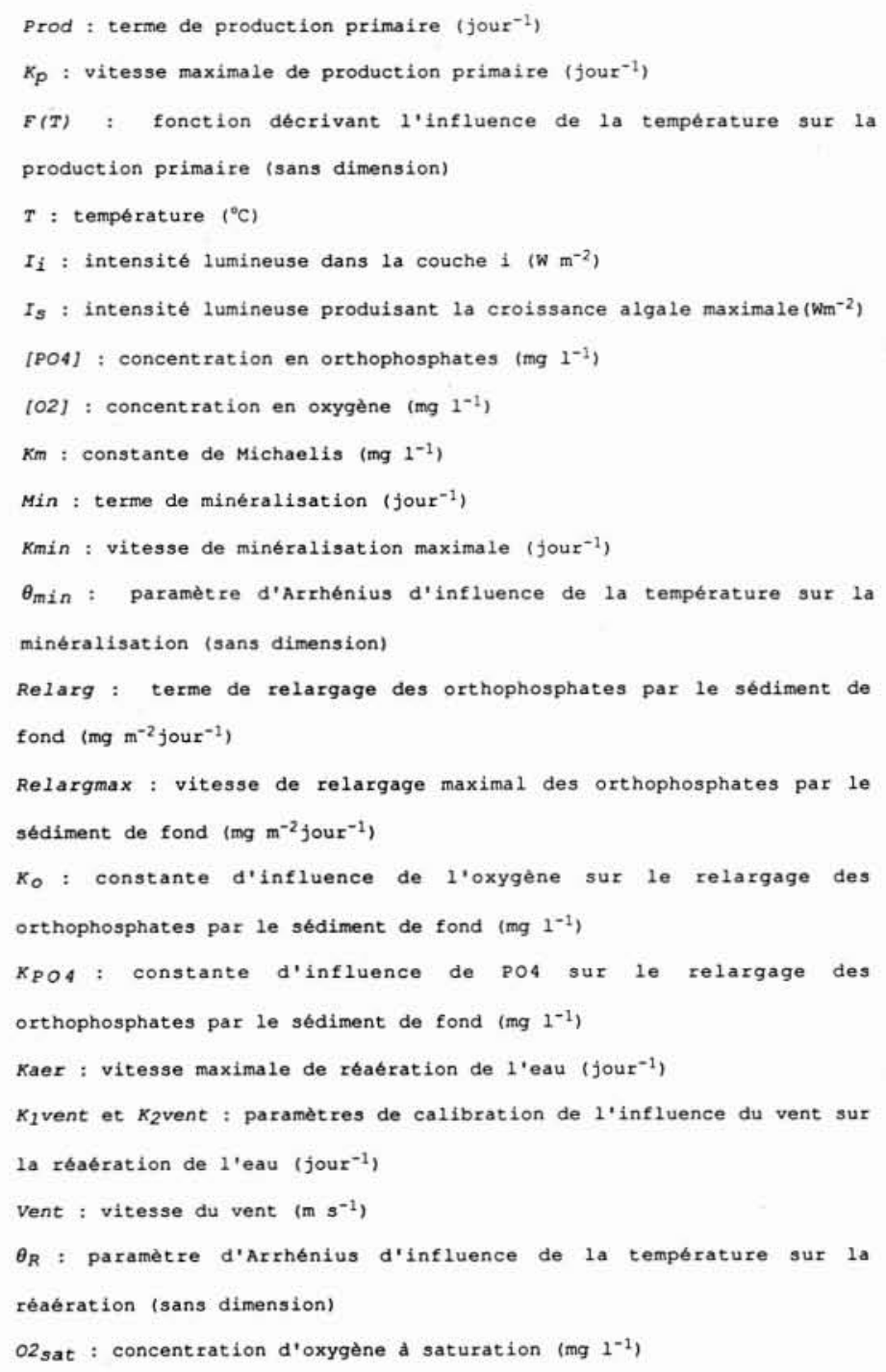




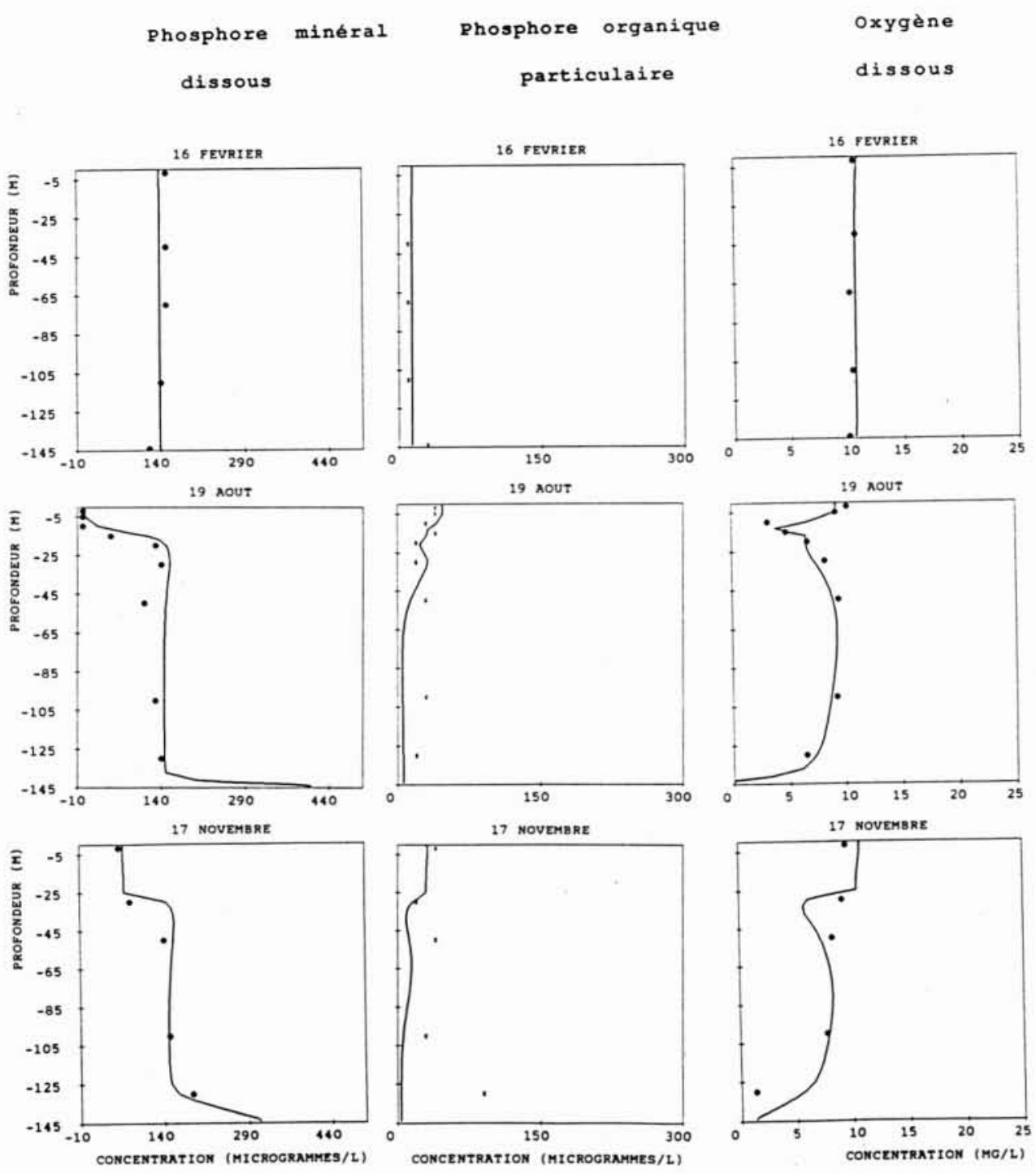

2. Profils de concentration d'orthophosphates, de phosphore particulaire et d'oxygène en quelques dates de l'année 1981.

Les valeurs des paramètres sont présentées dans le tableau 2. La figure 2 montre les profils de concentration calculés par le modèle en quelques dates significatives du cycle annuel du lac.

Ces résultats montrent que les principales caractéristiques du cycle du phosphore dans le lac du Bourget sont correctement représentées.

La diminution des concentrations d'orthophosphates dans les couches superficielles du lac, au printemps, coïncide avec l'augmentation de la production primaire qui se traduit par l'augmentation des concentrations de phosphore organique particulaire.

L'évolution de la biomasse phytoplanctonique repré- sentée par le phosphore organique particulaire est en assez bon accord avec les mesures réalisées. La représentation de la biomasse phytoplanctonique par le phosphore particulaire constitue l'hypothèse forte du modèle biogéochimique et n'est probablement pas vérifiée tout au long de l'année. De plus, les résultats du modèle sont comparés avec des " mesures " de phosphore particulaire qui, en fait, sont calculées par différence entre la concentration de phosphore total et d'orthophosphates. Ainsi, ces valeurs peuvent inclure, à certaines périodes de l'année, une fraction de phosphore particulaire d'origine allogène et, par ailleurs, ne tiennent pas compte de la fraction du phosphore organique dissous. 


\subsection{Sous-modèle Oxygène}

La production primaire produit de l'oxygène et en contrepartie la minéralisation du Phosphore organique particulaire en consomme. Un même rapport stœchiométrique est utilisé pour calculer l'oxygène produit ou consommé.

La réoxygénation du lac en surface (éq. 6) est fonction du coefficient de réaération qui dépend de la vitesse du vent et de la différence entre la concentration d'oxygène à saturation et la concentration existante (GAILLARD, 1981). La concentration d'oxygène à saturation est calculée par la formule de Truesdale (1955).

L'oxygène est consommé par la demande benthique du sédiment selon un taux constant.

\subsubsection{Calibration du sous-modèle Oxygène}

Dans le modèle Phosphore + Oxygène, nous avons repris les paramètres associés au cycle du phosphore, obtenus lors de la calibration du sous-modèle Phosphore. Les paramètres spécifiques du cycle de l'oxygène sont présentés dans le tableau 3.

La valeur du rapport Oxygène-Phosphore est estimée à partir de l'équation classique proposée par REDFIELD (1963) pour la minéralisation aérobie du phytoplancton marin :

$\mathrm{C}_{106} \mathrm{H}_{263} \mathrm{O}_{110} \mathrm{~N}_{16} \mathrm{P}+138 \mathrm{O}_{2}=$

$$
=106 \mathrm{CO}_{2}+122 \mathrm{H}_{2} \mathrm{O}+16 \mathrm{HNO}_{3}+\mathrm{H}_{3} \mathrm{PO}_{4} \text {. }
$$

D'après cette équation, la minéralisation d'un atome de phosphore organique consomme 276 atomes d'oxygène

Tableau 2. Valeurs des paramètres du sousmodèle Phosphore.

$\begin{array}{ll}\text { Kp } & : 0,700 \mathrm{j}^{-1} \\ \text { Is } & : 80 \mathrm{~W} / \mathrm{m}^{2} \\ K m & : 0,010 \mathrm{mg} / 1 \\ \text { Kmin } & : 0,080 \mathrm{j}^{-1} \\ \text { Omin } & : 1,050 \\ \text { Relargmax } & : 0,100 \mathrm{~g} / \mathrm{m} 2 / \mathrm{j} \\ \text { KPO4 } & : 0,150 \mathrm{mg} / 1 \\ \text { Ko } & : 1,700 \mathrm{mg} / 1 \\ \text { Vhypo } & : 0,300 \mathrm{~m} / \mathrm{j} \\ \text { Vépi } & : 0,150 \mathrm{~m} / \mathrm{j}\end{array}$

Tableau 3. Valeurs des paramètres du sousmodèle Oxygène.

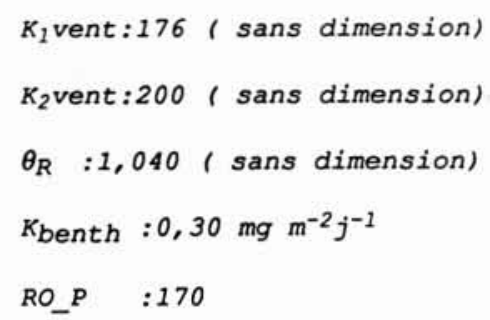

soit $142 \mathrm{mg}$ d'oxygène consommés pour $1 \mathrm{mg}$ de phosphore minéralisé. La valeur obtenue par calibration est légèrement supérieure à cette valeur théorique.

Les profils de concentration de phosphore minéral dissous et de phosphore organique particulaire (fig. 2) sont les mêmes pour les deux simulations, à l'exception des concentrations d'orthophosphates dans l'hypolimnion puisque, dans la première simulation, l'oxygène, qui influe sur le relargage des orthophosphates par le sédiment, est une variable de forçage du modèle alors que, dans la deuxième simulation, les concentrations d'oxygène sont calculées par le modèle.

Dans la première simulation, l'absence de mesures de concentration d'oxygène sur le fond du lac (la plus proche mesure est à $15 \mathrm{~m}$ au-dessus du fond) conduit à une sous-estimation du relargage, les concentrations d'oxygène diminuant très rapidement au voisinage du fond. Le modèle Phosphore + Oxygène donne probablement des valeurs plus réalistes des concentrations d'orthophosphates au voisinage du fond du lac.

L'évolution saisonnière des concentrations d'oxygène dans le lac du Bourget présente des profils caractéristiques que le modèle parvient à reproduire correctement (fig. 2).

La production primaire printanière entraîne une augmentation des concentrations d'oxygène dans les couches de surface, avec une sursaturation sous la surface pendant la période estivale.

Dès la fin du printemps, la consommation d'oxygène dans le métalimnion s'accentue et cause un déficit d'oxygène qui s'accroît de façon très marquée durant l'été.

Dans l'hypolimnion, la consommation d'oxygène est contrôlée par la demande benthique dont l'influence augmente quand on approche du fond du lac. 


\section{Conclusion}

Le modèle thermique et biogéochimique présenté décrit de façon satisfaisante l'évolution saisonnière des variables Température, Phosphore et Oxygène dans le lac du Bourget.

Le modèle thermique, calibré d'après les données de 1981 et vérifié sur une période longue de 8 années (19761983) parvient à simuler les variations thermiques interannuelles dans le lac.

La formulation de la dispersion, précédemment utilisée dans la modélisation du lac Léman (TASsıN, 1986) confirme sa validité. Cette paramétrisation confère son originalité au modèle par la spécificité de l'expression du coefficient de dispersion dans l'épilimnion, le métalimnion et l'hypolimnion.
L'accord avec les valeurs et les profils verticaux des coefficients de dispersion rencontrés sur d'autres lacs permet de penser que les coefficients calculés par le modèle représentent de façon correcte les phénomènes de transport de chaleur dans le lac.

Le modèle de qualité de l'eau associé au modèle thermique utilise, pour représenter le transport dispersif des éléments dissous, les coefficients de dispersion obtenus dans le calcul du transport de chaleur.

Les résultats de l'année 1981 montrent que le modèle est capable de simuler les principales caractéristiques du cycle annuel du phosphore et de l'oxygène. La vérification du modèle biogéochimique doit être réalisée avec une série de données indépendantes.

\section{Références bibliographiques}

Chahuneau F., Des Clers S., Meyer J. A. (1980). - Les modèles de simulation en écologie lacustre, Présentation des différentes approches et analyse des modèles existants. Acta Oecologica, Oecologica Generalis ; 1, 1, p. $27-50$.

Chahuneau F., Des Clers S., Meyer J. A. (1983). Analysis of prediction uncertainty : Monte Carlo simulation and nonlinear least-squares estimation of a vertical transport submodel for lake Nantua. In « Uncertainty and forecasting of water quality ", M. B. Beck and G. Van Straten (Ed.), Springer-Verlag, 183-203.

Chahuneau F. (1984). - Modélisation thermique saisonnière du lac de Nantua. Verh. Internat. Limnol., 22, 115-131.

GAILLARD J. (1981). - A predictive model for water quality in reservoirs and its application to selective withdrawal, $\mathrm{Ph}$. D. thesis, Colorado State University, 232 p.

GrifFin T. T., Ferrara R. A. (1984). - A multicomponent model of phosphorus dynamics in reservoirs. Water Resour. Bul., 20, 5, 777-788.

IMBODEN D. M. (1974). - Phosphorus model of lake eutrophication. Limnol. Oceanogr., 19, 297-304.
IMboden D. M., GÄChter R. (1978). - A dynamic lake model for trophic state prediction. Ecol. Modelling, 4, 77-98.

Jaquet J. M., Faverger P. Y., Peter A., Vernet J. P. (1983). - Premières données sur la matière en suspension dans le Léman. Rapport interne, Institut Forel, Université de Genève ( 83 p.).

Redfield A. C., Ketchum B. H., Richards F. A. (1963). The influence of organisms on the composition of sea water, p. 26-77, In M. H. Hill [ed.], The sea, vol. 2. Wiley-Interscience.

TASsin B. (1986). - Modélisation hydrodynamique, thermique et biogéochimique du lac Léman. Thèse de Doctorat de l'Ecole nationale des Ponts et Chaussées, Paris (250 p.).

TASsin B. (1987). - Un modèle de l'évolution saisonnière du phosphore dans le lac Léman. Sciences de l'eau, 6, 67-95.

Truesdale G. A., Downing A. L., Lowden G. F. (1955). The solubility of oxygen in pure water and sea-water. $J$. Appl. Chem., 5, p. 53-62.

Vinçon-Leite B., TAssin B., Mouchel J. M. (1989). Modélisation de l'évolution thermique saisonnière du lac du Bourget (France). Revue des sciences de l'eau, vol. 2, $\mathrm{n}^{*}$ 4, 483-510. 